RU99-07-B

NYU-TH/99/07/01

CERN-TH/99-195

hep-th/9909012

\title{
SUPERSYMMETRY AND GRAVITATIONAL QUADRUPOLES
}

\author{
Ioannis Giannakis ${ }^{a}$, James T. Liu ${ }^{a, d}$ and Massimo Porrati ${ }^{a, b, c} \dagger$ \\ (a) Physics Department, The Rockefeller University \\ 1230 York Avenue, New York, NY 10021-6399 \\ (b) Department of Physics, New York University \\ 4 Washington Pl., New York, NY 10003 \\ (c) Theory Division, CERN \\ CH 1211 Geneva 23 (Switzerland) \\ (d) Randall Laboratory of Physics, University of Michigan \\ Ann Arbor, MI 48109
}

\begin{abstract}
We derive model independent, non-perturbative supersymmetric sum rules for the gravitational quadrupole moments of arbitrary-spin particles in any $N=1$ supersymmetric theory. These sum rules select a "preferred" value of $h=1$ where the " $h$-factor" is the gravitational quadrupole analog of the gyromagnetic ratio or $g$-factor. This value of $h=1$ corresponds identically to the preferred field theory value obtained by tree-level unitarity considerations. The presently derived $h$-factor sum rule complements and generalizes previous work on electromagnetic moments where $g=2$ was shown to be preferred by both supersymmetric sum rule and tree-level unitarity arguments.
\end{abstract}

\footnotetext{
$\dagger$ e-mail: giannak@theory.rockefeller.edu,jimliu@umich.edu, massimo.porrati@nyu.edu
} 


\section{Introduction.}

In models with unbroken supersymmetry, it is often the case that the nature of the supersymmetry algebra leads to many powerful results on the structure of the theory itself. Such results often take the form of model independent sum rules that must be obeyed by the states in the supersymmetric spectrum. For example, the added constraint of unbroken supersymmetry requires that the gyromagnetic ratio of the electron (as the spin- $1 / 2$ member of a chiral multiplet) is given exactly by $g=2$ [1]. Such results have since been generalized to yield constraints on the electromagnetic multipole moments of any massive particle state in $N=1[2,3]$ and $N=2$ [4] supermultiplets. These sum rules were derived using general principles and hold for any electromagnetic current commuting with supersymmetry.

In this letter we apply similar techniques to the study of gravitational interactions in a theory with unbroken supersymmetry and derive sum rules for the gravitational quadrupole moments of the particle states in arbitrary $N=1$ massive supermultiplets. Following [5], we define an " $h$-factor", which is the gravitational analog of the gyromagnetic ratio. The $h$-factors of the different states in a massive $N=1$ supermultiplet are no longer arbitrary, but are related through the gravitational quadrupole sum rule; they are determined in terms of a single real quantity (which may be taken as the $h$-factor of the central member of the supermultiplet). Furthermore, supersymmetry selects a "natural" value of $h=1$, which in addition has the property of yielding tree-level unitarity up to the Planck scale for gravitational interactions $[6,7,5]$. In this sense, the $h$-factor sum rule and its consequences resemble those obtained for the usual gyromagnetic ratio, where the preferred value of $g=2$ was similarly selected by both tree-level unitarity $[8,9]$ and supersymmetric sum rule [2] considerations (for yet another reason, see [10]).

Indeed our derivation of the $h$-factor sum rules follows the method of [2], and involves the transformation properties of a conserved stress tensor $T_{\mu \nu}$ that commutes with the $N=1$ supersymmetry algebra. The main complication in obtaining the sum rules, that was not present in [2], is the requirement of working to second order in the momentum transfer in the appropriate matrix elements. This, however, has been addressed in [3], where a generalization to arbitrary electromagnetic multipole moments was presented.

Although the procedure used in obtaining the sum rules is by now standard, care must be taken in manipulating the supersymmetry algebra since various factors from different sources have to be combined in a precise manner. We follow the conventions for the Dirac algebra and $N=1$ supersymmetry given in [3]. Thus we start with the $N=1$ algebra written in the form $\left\{Q_{\alpha}, \bar{Q}_{\beta}\right\}=2\left(\gamma^{\mu}\right)_{\alpha \beta} P_{\mu}$, so that in the rest frame of a massive single particle state, we have

$$
\left\{Q_{\frac{1}{2}}^{L}, Q_{-\frac{1}{2}}^{R}\right\}=2 M, \quad\left\{Q_{-\frac{1}{2}}^{L}, Q_{\frac{1}{2}}^{R}\right\}=2 M
$$


Note that the superscripts $L, R$ denote chiralities,

$$
\gamma_{5} Q^{L}= \pm Q^{L}
$$

whereas the subscripts $\pm \frac{1}{2}$ denote helicities,

$$
i \gamma^{12} Q_{ \pm \frac{1}{2}}= \pm Q_{ \pm \frac{1}{2}}
$$

After proper normalization, the supersymmetry algebra, (1), corresponds to a Clifford algebra with two fermionic degrees of freedom. Thus irreducible massive $N=1$ representations are generated by acting with creation operators $Q_{ \pm \frac{1}{2}}^{R}$ on a superspin- $j$ Clifford vacuum, $|j\rangle$, that is annihilated by $Q_{ \pm \frac{1}{2}}^{L}$. As a result, this generates the massive $N=1$ superspin- $j$ multiplet with spins $\left(j+\frac{1}{2}, j, j, j-\frac{1}{2}\right)$.

Since the supercharges $Q_{ \pm \frac{1}{2}}^{L, R}$ are operators of spin $1 / 2$, we use a shorthand notation of labeling the spin- $j$ Clifford vacuum by $|0\rangle$, along with the superpartner states $|\uparrow\rangle,|\downarrow\rangle$ and $|\uparrow\rangle$, which are generated by (normalized) $Q_{\frac{1}{2}}^{R}, Q_{-\frac{1}{2}}^{R}$ and $Q_{\frac{1}{2}}^{R} Q_{-\frac{1}{2}}^{R}$, respectively. While this notation is initially convenient, it only addresses the action of the supercharges on the Clifford vacuum. The actual states in the multiplet are only obtained after taking the Clebsch-Gordan combination of angular momenta between the spin- $j$ Clifford vacuum and the spins $[(1 / 2)+2(0)]$, corresponding to $[(|\uparrow\rangle,|\downarrow\rangle)+|0\rangle+|\uparrow\rangle]$.

Since the gravitational quadrupole moment is defined in terms of moments of the stress tensor, we proceed with an examination of the multiplet containing $T_{\mu \nu}$. For a superconformal theory, this is the multiplet of currents $\left(T_{\mu \nu}, j_{\mu \alpha}, j_{\mu}^{(5)}\right)$, where $\alpha$ is a spinor index. More generally, theories invariant under $N=1$ supersymmetry may nevertheless violate dilatation invariance, $T_{\mu}^{\mu} \neq 0$, supersymmetric invariance, $\gamma^{\mu} j_{\mu} \neq 0$, or $R$-invariance, $\partial^{\mu} j_{\mu}^{(5)} \neq 0$, or any combination of these symmetries.

We first consider the case of ordinary $N=1$ supersymmetry, without conformal invariance, but with a conserved $R$-current:

$$
T_{\mu}^{\mu} \neq 0, \quad \gamma^{\mu} j_{\mu} \neq 0, \quad \partial^{\mu} j_{\mu}^{(5)}=0 .
$$

The resulting multiplet of currents contains $\left(j_{\mu}^{(5)}, j_{\mu}, T_{\mu \nu}, t_{\mu \nu}\right)$, where $t_{\mu \nu}$ is a conserved antisymmetric tensor, and has the following transformation properties:

$$
\begin{aligned}
\delta j_{\mu}^{(5)} & =i \bar{\epsilon} \gamma_{5} j_{\mu} \\
\delta j_{\mu} & =\left[-2 i \gamma^{\nu}\left(T_{\mu \nu}+t_{\mu \nu}\right)-\gamma_{5} \gamma^{\lambda} \partial_{\lambda} j_{\mu}^{(5)}-\frac{i}{2} \epsilon_{\mu \nu \lambda \sigma} \gamma^{\nu} \partial^{\lambda} j^{\sigma(5)}\right] \epsilon, \\
\delta T_{\mu \nu} & =-\frac{1}{2} \bar{\epsilon} \gamma_{(\mu}{ }^{\lambda} \partial_{\lambda} j_{\nu}, \\
\delta t_{\mu \nu} & =-\frac{i}{4} \epsilon_{\mu \nu \lambda \sigma} \bar{\epsilon} \gamma_{5} \gamma^{\sigma} \gamma^{\rho} \partial^{\lambda} j_{\rho} .
\end{aligned}
$$


We find that $\left[\delta_{1}, \delta_{2}\right] O=-2 i\left(\bar{\epsilon}_{2} \gamma^{\lambda} \epsilon_{1}\right) \partial_{\lambda} O$ where $O=\left(j_{\mu}^{(5)}, j_{\mu}, T_{\mu \nu}, t_{\mu \nu}\right)$, matching our normalization of the $N=1$ algebra. It follows then that two successive supersymmetry transformations on the conserved current, $T_{\mu \nu}$, gives

$$
\begin{aligned}
\delta_{\eta} \delta_{\epsilon} T_{\mu \nu}= & i \bar{\epsilon} \gamma_{(\mu}{ }^{\lambda \rho} \eta \partial_{\lambda}\left(T_{\nu) \rho}+t_{\nu) \rho}\right)-i \bar{\epsilon} \gamma^{\lambda} \eta \partial_{\lambda} T_{\mu \nu} \\
& +\frac{3}{4} \bar{\epsilon} \gamma_{5} \gamma^{\lambda} \eta\left(\square \eta_{\lambda(\mu}-\partial_{\lambda} \partial_{(\mu}\right) j_{\nu)}^{(5)}-\frac{1}{4} \bar{\epsilon} \gamma_{5} \gamma^{\lambda} \eta\left(\square \eta_{\mu \nu}-\partial_{\mu} \partial_{\nu}\right) j_{\lambda}^{(5)} .
\end{aligned}
$$

The matrix elements of this equation between single particle states which belong to the same $N=1$ multiplet give rise to sum rules for the gravitational multipoles of the particle states.

The gravitational moments may be obtained by performing a multipole expansion of the matrix elements $\left\langle j^{\prime}, m^{\prime}, \vec{p}\left|T_{\mu \nu}\right| j, m, 0\right\rangle$ of the stress tensor. While rotational symmetry allows the matrix elements to be decomposed in terms of spherical tensors (see e.g. [11]) or cartesian tensors of definite angular momentum [12], we find it sufficient to take a general expansion with indefinite angular momentum terms:

$$
\begin{aligned}
\left\langle j^{\prime}, m^{\prime}, \vec{p}\left|T_{00}\right| j, m, 0\right\rangle & =\sum_{l=0}^{\infty} \frac{1}{l !}(i p)_{i_{1}}(i p)_{i_{2}} \cdots(i p)_{i_{l}}\left\langle j^{\prime}, m^{\prime}, 0,\left|M_{i_{1} i_{2} \cdots i_{l}}^{(l)}\right| j, m, 0\right\rangle \\
& =\left\langle 0\left|M^{(0)}\right| 0\right\rangle+i p^{i}\left\langle 0\left|M_{i}^{(1)}\right| 0\right\rangle-\frac{1}{2 !} p^{i} p^{j}\left\langle 0\left|M_{i j}^{(2)}\right| 0\right\rangle+\cdots, \\
\left\langle j^{\prime}, m^{\prime}, \vec{p}\left|T_{0 i}\right| j, m, 0\right\rangle & =\sum_{l=0}^{\infty} \frac{1}{(l+1) !}(i p)_{i_{1}}(i p)_{i_{2}} \cdots(i p)_{i_{l}}\left\langle j^{\prime}, m^{\prime}, 0,\left|N_{i i_{1} \cdots i_{l}}^{(l+1)}\right| j, m, 0\right\rangle \\
& =\left\langle 0\left|N_{i}^{(1)}\right| 0\right\rangle+\frac{i}{2 !} p^{j}\left\langle 0\left|N_{i j}^{(2)}\right| 0\right\rangle-\frac{1}{3 !} p^{j} p^{k}\left\langle 0\left|N_{i j k}^{(3)}\right| 0\right\rangle+\cdots, \\
\left\langle j^{\prime}, m^{\prime}, \vec{p}\left|T_{i j}\right| j, m, 0\right\rangle & =\sum_{l=0}^{\infty} \frac{1}{(l+2) !}(i p)_{i_{1}}(i p)_{i_{2}} \cdots(i p)_{i_{l}}\left\langle j^{\prime}, m^{\prime}, 0,\left|L_{i j i_{1} \cdots i_{l}}^{(l+2)}\right| j, m, 0\right\rangle \\
& =\frac{1}{2 !}\left\langle 0\left|L_{i j}^{(2)}\right| 0\right\rangle+\frac{i}{3 !} p^{k}\left\langle 0\left|L_{i j k}^{(3)}\right| 0\right\rangle-\frac{1}{4 !} p^{k} p^{l}\left\langle 0\left|L_{i j k l}^{(4)}\right| 0\right\rangle+\cdots,
\end{aligned}
$$

where some of the quantum numbers $\left(j^{\prime}, m^{\prime}\right)$ and $(j, m)$ have been suppressed. Note that the first index of $N^{(l+1)}$ and the first two indices of $L^{(l+2)}$ are distinct from the remaining ones, and do not share any definite symmetry properties.

To $p^{2}$ order, conservation of the stress tensor, $p^{\mu} T_{\mu \nu}=0$, provides us with the following relations:

$$
\begin{aligned}
& M^{(0)}+i M \frac{p^{i} p^{j}}{p^{2}} N_{i j}^{(2)}=0, \quad N_{i}^{(1)}=0, \quad i p^{i}\left[M_{i}^{(1)}-\frac{i}{2 M} N_{i}^{(1)}+\frac{i M}{3} \frac{p^{j} p^{k}}{p^{2}} N_{i j k}^{(3)}\right]=0, \\
& \frac{1}{2} p^{i} p^{j}\left[M_{i j}^{(2)}-\frac{i}{2 M} N_{i j}^{(2)}+\frac{i M}{6} \frac{p^{k} p^{l}}{p^{2}} N_{i j k l}^{(4)}\right]=0, \quad N_{i}^{(1)}+\frac{i M}{3} \frac{p^{j} p^{k}}{p^{2}} L_{i j k}^{(3)}=0, \\
& p^{i}\left[i N_{j i}^{(2)}+\frac{1}{2 M} L_{i j}^{(2)}-\frac{M}{6} \frac{p^{k} p^{l}}{p^{2}} L_{i j k l}^{(4)}\right]=0, \quad p^{j} L_{i j}^{(2)}=0 .
\end{aligned}
$$


By multiplying the first and penultimate equations by $p^{2}$, and by differentiating with respect to the momentum, we find the relations

$$
N_{(i j)_{S}}^{(2)}=i \frac{M^{(0)}}{M} \delta_{i j}, \quad L_{i(j k l)_{S}}^{(4)}=-2 \frac{M^{(0)}}{M^{2}}\left(\delta_{i j} \delta_{k l}+\delta_{i k} \delta_{j l}+\delta_{i l} \delta_{j k}\right),
$$

where ()$_{S}$ denotes complete symmetrization with weight one.

Note that the gravitational dipole term in the expansion of $T_{00}, M_{i}^{(1)}$, can be removed by translating $T_{00}$ and choosing the translation $d_{i}$ appropriately, i.e.:

$$
\begin{aligned}
\left\langle\vec{p}\left|T_{00}(\vec{x}+\vec{d})\right| 0\right\rangle & =\left\langle\vec{p}\left|\exp \left(i d_{i} p^{i}\right) T_{00}(x)\right| 0\right\rangle=\left\langle\vec{p}\left|\left(1+i d_{i} p^{i}+. .\right) T_{00}\right| 0\right\rangle \\
& =\left\langle\vec{p}\left|T_{00}\right| 0\right\rangle+i M^{(0)} d_{i} p^{i}+O\left(p^{2}\right)=M^{(0)}+i p^{i} M_{i}^{(1)}+i M^{(0)} d_{i} p^{i}+\cdots
\end{aligned}
$$

Therefore there is no physical content in $M_{i}^{(1)}$, and we take it to vanish henceforth.

It was demonstrated in [5] that, outside of the Newtonian limit, the nonrelativistic definition of the gravitational quadrupole has to be amended. The general expression for the quadrupole moment (in $D=4$ ) is [5]:

$$
Q^{i j}=\int d^{3} x\left(x^{i} x^{j}-\frac{1}{3} \delta^{i j} x^{2}\right) \mathcal{T}_{00}
$$

where $\mathcal{T}_{00}=T_{00}+\delta^{i j} T_{i j}$. By writing $\mathcal{T}_{00}(x)=\int \frac{d^{3} p}{(2 \pi)^{3}} \overline{\mathcal{T}}_{00}(p) e^{-i p x}$, we find that

$$
\begin{aligned}
Q_{i j} & =\left.\left[\left(i \frac{\partial}{\partial p^{i}}\right)\left(i \frac{\partial}{\partial p^{j}}\right)-\frac{1}{3} \delta_{i j}\left(i \frac{\partial}{\partial p^{k}}\right)\left(i \frac{\partial}{\partial p_{k}}\right)\right] \overline{\mathcal{T}}_{00}\right|_{p=0} \\
& =M_{i j}^{(2)}-\frac{1}{3} \delta_{i j} M_{k}^{(2) k}+\frac{1}{12} \delta^{k l} L_{i j k l}^{(4)}-\frac{1}{36} \delta_{i j} \delta^{a b} \delta^{k l} L_{a b k l}^{(4)}
\end{aligned}
$$

Similarly the definitions for the mass $M=\int d^{3} x T_{00}$ and the angular momentum $J_{i}=$ $\int d^{3} x \epsilon_{i j k} x^{j} T^{0 k}$ provide us with the relations $M=M^{(0)}$ and $J_{i}=-\frac{1}{2} \epsilon_{i j k} N^{(2) j k}$. The relevant expansions for the matrix elements of $\mathcal{T}_{00}$ and $T_{0 i}$ then become

$$
\begin{aligned}
\left\langle j^{\prime}, m^{\prime}, \vec{p}\left|\mathcal{T}_{00}\right| j, m, 0\right\rangle & =M_{j} \delta_{j j^{\prime}} \delta_{m m^{\prime}}-\frac{1}{2} p^{i} p^{j}\left\langle j^{\prime}, m^{\prime}, 0\left|Q_{i j}\right| j, m, 0\right\rangle+\cdots, \\
\left\langle j^{\prime}, m^{\prime}, \vec{p}\left|T_{0 i}\right| j, m, 0\right\rangle & =\frac{i}{2} p^{j} \delta_{j j^{\prime}} \delta_{m m^{\prime}}+\frac{i}{2} \epsilon_{i j k} p^{j}\left\langle j^{\prime}, m^{\prime}, 0\left|J^{k}\right| j, m, 0\right\rangle+\cdots,
\end{aligned}
$$

where we have omitted trace terms (i.e. terms proportional to the contracted momentum $\left.p^{2}\right)$. 
As in the electromagnetic case [3], general multipole moment sum rules are derived by taking the double supersymmetry variation of the conserved stress tensor $T_{\mu \nu}$,

$$
\begin{aligned}
\delta_{\eta} \delta_{\epsilon} T_{\mu \nu} & =\left[\bar{\eta} Q,\left[\bar{\epsilon} Q, T_{\mu \nu}\right]\right] \\
& =\bar{\eta} Q \bar{\epsilon} Q T_{\mu \nu}-\bar{\eta} Q T_{\mu \nu} \bar{\epsilon} Q-\bar{\epsilon} Q T_{\mu \nu} \bar{\eta} Q+T_{\mu \nu} \bar{\epsilon} Q \bar{\eta} Q
\end{aligned}
$$

and evaluating it between single particle states $\langle\alpha|$ and $|\beta\rangle$. Since $\delta_{\eta} \delta_{\epsilon} T_{\mu \nu}$ is known, and is given by (6), the right hand side of (14) then relates the matrix elements of $T_{\mu \nu}$ among the superpartner states generated by $Q$. By choosing the initial and final states to be the superspin- $j$ Clifford vacuum, and by picking $\eta_{L}=0$, we find

$$
\left\langle\alpha, \vec{p}\left|\delta_{\eta_{R}} \delta_{\epsilon} T_{\mu \nu}\right| \beta, 0\right\rangle=\left\langle\alpha, \vec{p}\left|T_{\mu \nu} \bar{\epsilon} Q \bar{\eta}_{R} Q\right| \beta, 0\right\rangle-\left\langle\alpha, 0\left|\bar{\epsilon} Q^{(p)} L^{-1}(\vec{p}) T_{\mu \nu} \bar{\eta}_{R} Q\right| \beta, 0\right\rangle,
$$

where $Q^{(p)}$ denotes the Lorentz boost of $Q$, namely $Q^{(p)}=L^{-1}(\vec{p}) Q L(\vec{p})$, and $|\alpha, \vec{p}\rangle=$ $L(\vec{p})|\alpha, 0\rangle$. This expression provides the basis of determining both the "vanishing" and "diagonal" sum rules.

For the vanishing sum rule, we set $\epsilon_{L}=0$ so that only $\epsilon_{R}$ is active. Since Eq. (6) indicates that $\delta_{\eta_{R}} \delta_{\epsilon_{R}} T_{\mu \nu}=0$, the sum rule (15) becomes

$$
\left\langle\alpha, \vec{p}\left|T_{\mu \nu} \bar{\epsilon}_{R} Q \bar{\eta}_{R} Q\right| \beta, 0\right\rangle=0
$$

This demonstrates that all matrix elements of the stress tensor vanish between states $|0\rangle$ and $|\uparrow\rangle$, and hence that there are no off-diagonal moments between the two spin- $j$ states of the supermultiplet.

For the diagonal sum rule, we choose $\epsilon_{R}=0$ and find instead

$$
\begin{aligned}
& \left\langle\alpha, 0\left|\bar{\epsilon}_{L} Q L^{-1}(\vec{p}) T_{\mu \nu} \bar{\eta}_{R} Q\right| \beta, 0\right\rangle=2 M\left(\bar{\epsilon}_{L} \gamma^{0} \eta_{R}\right)\left\langle\alpha, \vec{p}\left|T_{\mu \nu}\right| \beta, 0\right\rangle \\
& -\left\langle\alpha, \vec{p}\left|\delta_{\eta_{R}} \delta_{\epsilon_{L}} T_{\mu \nu}\right| \beta, 0\right\rangle-\left\langle\alpha, 0\left|\left[L^{-1}(\vec{p}), \bar{\epsilon}_{L} Q\right] T_{\mu \nu} \bar{\eta}_{R} Q\right| \beta, 0\right\rangle
\end{aligned}
$$

The first line of (17) essentially relates the matrix elements in the superpartner state with those in the original state, while the second line provides correction terms originating from supersymmetry and from Lorentz boosts.

For the Lorentz boost correction, we make use of the fact that $Q$ transforms as a spinor to derive

$$
\begin{aligned}
{\left[L^{-1}(\vec{p}), \bar{\epsilon}_{L} Q\right] } & =\bar{\epsilon}_{L}\left(\sqrt{\frac{E+M}{2 M}}\left(1+\frac{p^{i}}{(E+M)} \gamma^{0 i}\right)-1\right) Q L^{-1}(\vec{p}) \\
& =\frac{p^{i}}{2 M} \bar{\epsilon}_{L} \gamma^{0 i} Q L^{-1}(\vec{p})+\cdots
\end{aligned}
$$


where the terms that have been dropped are all trace-like, and do not contribute to the static moments. Specializing to the gravitational quadrupole moment, we are interested in the supersymmetry variation $\delta_{\eta_{R}} \delta_{\epsilon_{L}} \mathcal{T}_{00}$. Using Eq. (6) we find that

$$
\begin{aligned}
\delta_{\eta_{R}} \delta_{\epsilon_{L}} T_{00}= & -p_{\lambda} \bar{\epsilon}_{L} \gamma_{0}{ }^{\lambda \rho} \eta_{R}\left(T_{0 \rho}+t_{0 \rho}\right)+p_{\lambda} \bar{\epsilon}_{L} \gamma^{\lambda} \eta_{R} T_{00} \\
& -\frac{3}{4} \bar{\epsilon}_{L} \gamma_{5} \gamma^{\lambda} \eta_{R}\left(\eta_{0 \lambda} p^{2}-p_{0} p_{\lambda}\right) j_{0}^{(5)}+\frac{1}{4} \bar{\epsilon}_{L} \gamma_{5} \gamma^{\lambda} \eta_{R}\left(\eta_{00} p^{2}-p_{0} p_{0}\right) j_{\lambda}^{(5)} \\
= & -p_{i}\left(\bar{\epsilon}_{L} \gamma_{0}{ }^{i j} \eta_{R}\right)\left(T_{0 j}+t_{0 j}\right)+p_{i}\left(\bar{\epsilon}_{L} \gamma^{i} \eta_{R}\right) T_{00}+O\left(p^{2}\right)
\end{aligned}
$$

and

$$
\begin{aligned}
\delta^{i j} \delta_{\eta_{R}} \delta_{\epsilon_{L}} T_{i j}= & -p_{\lambda} \bar{\epsilon}_{L} \gamma_{0}{ }^{\lambda \rho} \eta_{R}\left(T_{\rho 0}+t_{\rho 0}\right)+p_{\lambda} \bar{\epsilon}_{L} \gamma^{\lambda} \eta_{R} \delta^{i j} T_{i j}+p_{k} \bar{\epsilon}_{L} \gamma^{i k j} \eta_{R} t_{i j} \\
& -\frac{3}{4} \bar{\epsilon}_{L} \gamma_{5} \gamma^{\lambda} \eta_{R}\left(\eta_{i \lambda} p^{2}-p_{i} p_{\lambda}\right) j_{i}^{(5)}+\frac{1}{4} \bar{\epsilon}_{L} \gamma_{5} \gamma^{\lambda} \eta_{R}\left(\eta_{i i} p^{2}-p_{i} p_{i}\right) j_{\lambda}^{(5)} \\
= & -p_{i}\left(\bar{\epsilon}_{L} \gamma_{0}{ }^{i j} \eta_{R}\right)\left(T_{j 0}+t_{j 0}\right)+p_{k}\left(\bar{\epsilon}_{L} \gamma^{k} \eta_{R}\right) \delta^{i j} T_{i j}+O\left(p^{2}\right) .
\end{aligned}
$$

Note that the antisymmetric tensor $t_{\mu \nu}$ drops out when the two equations are combined:

$$
\delta_{\eta_{R}} \delta_{\epsilon_{L}} \mathcal{T}_{00}=-2 p_{i}\left(\bar{\epsilon}_{L} \gamma_{0}{ }^{i j} \eta_{R}\right) T_{0 j}+p_{i}\left(\bar{\epsilon}_{L} \gamma^{i} \eta_{R}\right) \mathcal{T}_{00}+O\left(p^{2}\right) .
$$

In particular, this is a consequence of the complete relativistically correct definition of the gravitational quadrupole moment, (11).

The diagonal sum rule now takes the form:

$$
\begin{aligned}
\frac{1}{2 M}\left\langle\alpha, 0\left|\bar{\epsilon}_{L} Q L^{-1}(\vec{p}) \mathcal{T}_{00} \bar{\eta}_{R} Q\right| \beta, 0\right\rangle= & \left(\bar{\epsilon}_{L} \gamma^{0} \eta_{R}\right)\left\langle\alpha, \vec{p}\left|\mathcal{T}_{00}\right| \beta, 0\right\rangle \\
& -i \epsilon^{i j k} \frac{p_{j}}{M}\left(\bar{\epsilon}_{L} \gamma_{k} \eta_{R}\right)\left\langle\alpha, \vec{p}\left|T_{0 i}\right| \beta, 0\right\rangle,
\end{aligned}
$$

where we have omitted terms of order $p^{2}$. Due to rotational invariance it is sufficient to examine the 33 component of the quadrupole moment. By substituting the definitions of the quadrupole and angular momentum matrix elements, (13), into Eq. (22) and by equating terms of the same order in momentum transfer, we arrive at the following diagonal sum rules:

$$
\begin{aligned}
\left\langle\uparrow\left|Q_{33}\right| \uparrow\right\rangle & =\left\langle 0\left|Q_{33}\right| 0\right\rangle+\frac{2}{3 M}\left\langle 0\left|J_{3}\right| 0\right\rangle, & & \left\langle\uparrow\left|Q_{33}\right| \downarrow\right\rangle=-\frac{1}{3 M}\left\langle 0\left|J_{-}\right| 0\right\rangle, \\
\left\langle\downarrow\left|Q_{33}\right| \downarrow\right\rangle & =\left\langle 0\left|Q_{33}\right| 0\right\rangle-\frac{2}{3 M}\left\langle 0\left|J_{3}\right| 0\right\rangle, & \left\langle\downarrow\left|Q_{33}\right| \uparrow\right\rangle & =-\frac{1}{3 M}\left\langle 0\left|J_{+}\right| 0\right\rangle .
\end{aligned}
$$

To derive the sum rules for the physical states of the superspin- $j$ multiplet, we add the supersymmetry generated spin $(|\uparrow\rangle$ or $|\downarrow\rangle)$ to the original spin using the appropriate Clebsch-Gordan combinations

$$
\begin{aligned}
& \left|j+\frac{1}{2}, m+\frac{1}{2}\right\rangle=\frac{1}{\sqrt{2 j+1}}[\sqrt{j+m+1}|j, m ; \uparrow\rangle+\sqrt{j-m}|j, m+1 ; \downarrow\rangle], \\
& \left|j-\frac{1}{2}, m+\frac{1}{2}\right\rangle=\frac{1}{\sqrt{2 j+1}}[-\sqrt{j-m}|j, m ; \uparrow\rangle+\sqrt{j+m+1}|j, m+1 ; \downarrow\rangle] .
\end{aligned}
$$


For a given state of spin $j$, the quadrupole moment is characterized by a single quantity, $Q^{(j)}$. By convention this is taken to be the matrix element of $Q_{33}$ in the $|j, m=j\rangle$ state. More generally, by the Wigner-Eckart theorem, we may write $(j \geq 1)$

$$
Q^{(j)}=\frac{j(2 j-1)}{3 m^{2}-j(j+1)}\left\langle\alpha, j, m\left|Q_{33}\right| \alpha, j, m\right\rangle .
$$

We now recall that the massive superspin- $j$ supermultiplet contains four states, of spins $\left(j+\frac{1}{2}, j, j, j-\frac{1}{2}\right)$. Using the vanishing sum rule and the ability to choose $|\uparrow\rangle$ as an alternate Clifford vacuum [2], we note that both spin- $j$ states have the same gravitational quadrupole moment. Thus for the four states there are three separate quantities, $\left(Q^{\left(j+\frac{1}{2}\right)}, Q^{(j)}, Q^{\left(j-\frac{1}{2}\right)}\right)$, which are related by the sum rules $(j \geq 3 / 2)$ :

$$
Q^{\left(j+\frac{1}{2}\right)}=Q^{(j)}+\frac{2 j}{3 M}, \quad Q^{\left(j-\frac{1}{2}\right)}=Q^{(j)} \frac{(j-1)(2 j+3)}{j(2 j+1)}-\frac{2}{3 M} \frac{(j-1)(2 j-1)}{(2 j+1)} .
$$

For $j=1 / 2$ and $j=1$ the sum rules simplify to

$$
Q^{(1)}=\frac{1}{3 M} \quad\left(j=\frac{1}{2}\right), \quad Q^{\left(\frac{3}{2}\right)}=Q^{(1)}+\frac{2}{3 M} \quad(j=1) .
$$

The gravitational quadrupole moment, $Q_{i j}$, may be related to an $h$-factor, which is the gravitational analog of the electromagnetic $g$-factor $[5,13,14,15]$. The $h$-factor may be defined in terms of the coupling of a spin- $j$ field $\varphi$ to the Riemann tensor that arises in the equations of motion as

$$
\left(\nabla_{\mu} \nabla^{\mu}-M^{2}\right) \varphi+h\left[R_{\mu \nu \lambda \sigma} \frac{1}{2} \Sigma^{\mu \nu} \frac{1}{2} \Sigma^{\lambda \sigma}\right] \varphi+\cdots=0
$$

where $\Sigma^{\mu \nu}$ is the Lorentz generator in the spin- $j$ representation. In the non-relativistic limit the Riemann coupling in (28) gives rise to a shift in energy

$$
\Delta E=-\frac{h}{2 M}\left[R_{i j l k} \frac{1}{2} \Sigma^{i j} \frac{1}{2} \Sigma^{k l}\right]=\frac{h}{2 M} J^{i} J^{j} \partial_{i} \partial_{j} \phi
$$

where we have used the fact that in four dimensions the components of the Riemann tensor are given in terms of the Newtonian potential $\phi$ as

$$
R_{i j k l}=\delta_{i k} \partial_{j} \partial_{l} \phi+\delta_{j l} \partial_{i} \partial_{k} \phi-\delta_{i l} \partial_{j} \partial_{k} \phi-\delta_{j k} \partial_{i} \partial_{l} \phi
$$

Note that, out of the original Lorentz generators, only the angular momentum generators $\Sigma^{i j}=\epsilon^{i j k} J_{k}$ remain in this limit.

The $h$-factor can now be related to the gravitational quadrupole $Q_{i j}$ by recalling that the change in energy of a particle in a static, slowly varying external Newtonian potential $\phi$ due to the quadrupole is

$$
\Delta E=\frac{1}{2} Q^{i j} \partial_{i} \partial_{j} \phi
$$


Comparing (29) with (31), we obtain the relation

$$
Q_{i j}=\frac{h}{M}\left(J_{i} J_{j}-\frac{1}{3} \delta_{i j} J^{2}\right),
$$

where the latter term is necessary to make $Q_{i j}$ traceless. Nevertheless this has no effect in Eq. (31) since $\partial_{i} \partial_{i} \phi=0$ in vacuum. Note the resemblance between the relations (32) for the $h$-factor and $\mu_{i}=\frac{g e}{2 M} J_{i}$ for the $g$-factor.

Combining the $h$-factor definition, (32), with the quadrupole matrix element, (25), we find $h_{j}=\frac{3 M}{j(2 j-1)} Q^{(j)}$ (where the quadrupole moment is only well defined for spins $\geq 1$ ). In terms of $h$-factors, the quadrupole sum rules, (26), now take on the final simpler form:

$$
\begin{gathered}
h_{j+\frac{1}{2}}=h_{j}-\frac{2\left(h_{j}-1\right)}{2 j+1}, \quad h_{j-\frac{1}{2}}=h_{j}+\frac{2\left(h_{j}-1\right)}{2 j+1} \quad\left(j \geq \frac{3}{2}\right), \\
h_{1}=1 \quad\left(j=\frac{1}{2}\right), \quad h_{\frac{3}{2}}=\frac{1}{3}\left(h_{1}+2\right) \quad(j=1) .
\end{gathered}
$$

The terms proportional to $\left(h_{j}-1\right)$ are related to a gravitational transition moment between the $j \pm \frac{1}{2}$ states of the multiplet, and vanish when $h=1$, yielding the possible value of $h=1$ for all members of the superspin- $j$ multiplet. This result from supersymmetry matches with those obtained previously $[7,14,15,5]$ that similarly suggest that $h=1$ is a natural value for the $h$-factor, in much the same way that $g=2$ is natural for the gyromagnetic ratio.

Finally we would like to extend our results to theories invariant under $N=1$ supersymmetry but violating $R$ symmetry. In this case the resulting multiplet of currents contains $\left(j_{\mu}^{(5)}, j_{\mu}, T_{\mu \nu}, P, Q\right)$, with the following transformation properties:

$$
\begin{aligned}
\delta j_{\mu}^{(5)} & =i \bar{\epsilon} \gamma_{5} j_{\mu}-\frac{i}{3} \bar{\epsilon} \gamma_{5} \gamma_{\mu} \gamma^{\rho} j_{\rho}, \\
\delta j_{\mu} & =\left[-2 i \gamma^{\lambda} T_{\mu \lambda}-\gamma_{5} \gamma^{\lambda} \partial_{\lambda} j_{\mu}^{(5)}+\gamma_{5} \gamma_{\mu} \partial^{\lambda} j_{\lambda}^{(5)}-\frac{i}{2} \epsilon \epsilon_{\mu \nu} \sigma \gamma^{\nu} \partial^{\lambda} j^{\sigma(5)}+\frac{1}{3} \gamma_{\mu \nu} \partial^{\nu}\left(P+i \gamma_{5} Q\right)\right] \epsilon, \\
\delta T_{\mu \nu} & =-\frac{1}{2} \bar{\epsilon} \gamma_{(\mu}{ }^{\lambda} \partial_{\lambda} j_{\nu)}, \\
\delta P & =-i \bar{\epsilon} \gamma^{\rho} j_{\rho}, \quad \delta Q=\bar{\epsilon} \gamma_{5} \gamma^{\rho} j_{\rho} .
\end{aligned}
$$

It follows then that two successive supersymmetry transformations on the conserved current $T_{\mu \nu}$ gives

$$
\begin{aligned}
\delta_{\eta} \delta_{\epsilon} T_{\mu \nu}= & i \bar{\epsilon} \gamma_{(\mu}^{\lambda \rho} \eta \partial_{\lambda} T_{\nu) \rho}-i \bar{\epsilon} \gamma^{\lambda} \eta \partial_{\lambda} T_{\mu \nu}+\frac{3}{4} \bar{\epsilon} \gamma_{5} \gamma^{\lambda} \eta\left(\square \eta_{\lambda(\mu}-\partial_{\lambda} \partial_{(\mu}\right) j_{\nu)}^{(5)} \\
& -\frac{1}{4} \bar{\epsilon} \gamma_{5} \gamma^{\lambda} \eta\left(\square \eta_{\mu \nu}-\partial_{\mu} \partial_{\nu}\right) j_{\lambda}^{(5)}+\frac{3}{4} \bar{\epsilon} \gamma_{5} \gamma_{\lambda} \eta\left(\eta_{\mu \nu} \eta^{\lambda \sigma}-\delta_{(\mu}^{\lambda} \delta_{\nu)}^{\sigma}\right) \partial_{\sigma} \partial^{\rho} j_{\rho}^{(5)} \\
& +\frac{1}{6} \bar{\epsilon} \eta\left(\square \eta_{\mu \nu}-\partial_{\mu} \partial_{\nu}\right) P+\frac{i}{6} \bar{\epsilon} \gamma_{5} \eta\left(\square \eta_{\mu \nu}-\partial_{\mu} \partial_{\nu}\right) Q .
\end{aligned}
$$


While added terms arise due to the non-conservation of $j_{\mu}^{(5)}$, they nevertheless do not contribute to the double variation of $\mathcal{T}_{00}$ to the order that would affect the quadrupole matrix elements. Thus $\delta_{\eta_{R}} \delta_{\epsilon_{L}} \mathcal{T}_{00}$ is unchanged from (21), and hence the resulting sum rules for the gravitational quadrupole moments coincide with those derived above for the $R$-invariant theory.

Although we have focused our attention on gravitational quadrupole moments, the sum rules we have derived may easily be generalized along the lines of [5] to encompass arbitrary gravitational multipoles. While such higher moments may lack clear physical significance, they many nevertheless provide a means for examining higher derivative couplings to gravity, especially in a string context. Additionally, the extension of the above to the case of extended supersymmetry should allow for the study of graviphoton sum rules in a model independent manner since the corresponding currents would indeed fall into the gravity sector of the theory.

\section{Acknowledgments.}

M.P. would like to thank the ITP at UCSB for its kind hospitality during completion of this work. This work was supported in part by the Department of Energy under Contract Number DE-FG02-91ER40651-TASK B, and by the NSF under grant PHY-9722083.

\section{References.}

[1] S. Ferrara and E. Remiddi, Phys. Lett. 53B (1974) 347.

[2] S. Ferrara and M. Porrati, Phys. Lett. B288 (1992) 85.

[3] I. Giannakis, J. T. Liu and M. Porrati, Phys. Rev. D58 (1998) 045016, hep-th/9803073.

[4] I. Giannakis and J. T. Liu, Phys. Rev. D58 (1998) 025009, hep-th/9711173.

[5] I. Giannakis, J. T. Liu and M. Porrati, Phys. Rev. D59 (1999) 104013, hep-th/9809142.

[6] M. Porrati, Phys. Lett. B304 (1993) 77.

[7] A. Cucchieri, M. Porrati and S. Deser, Phys. Rev. D51 (1995) 4543.

[8] S. Weinberg in Lectures on Elementary Particles and Quantum Field Theory, S. Deser, M. Grisaru and H. Pendleton, eds., MIT Press, Cambridge, MA (1970).

[9] S. Ferrara, M. Porrati and V.L. Telegdi, Phys. Rev. D46 (1992) 3529.

[10] R. Jackiw, Phys. Rev. D57 (1998) 2635.

[11] V. Rahal and H. C. Ren, Phys. Rev. D41 (1989) 1989.

[12] K.S. Thorne, Rev. Mod. Phys. 52 (1980) 299.

[13] I. B. Khriplovich, Sov. Phys. JETP 69 (1989) 217.

[14] I. B. Khriplovich and A. A. Pomeransky, Sov. Phys. JETP 86 (1998) 839, grqc/9710098.

[15] I. B. Khriplovich and A. A. Pomeransky, gr-qc/9809069. 